\title{
Malignant mesothelioma of the tunica vaginalis testis: A case report and literature review
}

\author{
NENG ZHANG, NI FU, SU PENG and XU LUO \\ Department of Urology, Affiliated Hospital of Zunyi Medical College, Zunyi, Guizhou 563003, P.R. China
}

Received August 9, 2017; Accepted October 11, 2017

DOI: $10.3892 / \mathrm{mco} .2017 .1450$

\begin{abstract}
Malignant mesothelioma of the tunica vaginalis testis is an extremely rare tumor without specific clinical manifestations, mainly including hydrocele formation and a painless mass. We herein present the case of a patient with hydrocele of the left testis, without any other complaints. Tunica vaginalis subinvolution was performed, and postoperative pathological examination revealed a malignant mesothelioma arising from the left tunica vaginalis testis. Whole-body positron emission tomography-computed tomography (PET-CT) and subsequent abdominal and pelvic magnetic resonance imaging (MRI) revealed no evidence of local lymphadenopathy. Radical left orchiectomy was performed after the pathological diagnosis. The pathological examination after the second surgery demonstrated that the tumor had invaded the adjacent periorchium and spermatic cord, but there was no evidence of local lymph node metastasis. Pemetrexed and cisplatin were administered at a dose of 900 and $130 \mathrm{mg}$, respectively, on the first day of a 28-day cycle. After 6 months of therapy, the disease had not progressed on abdominal and pelvic PET-CT and MRI. The patient was still followed up in our urology outpatient clinic at the time of the present report. Although testicular hydrocele is a common and easily diagnosed condition, detailed medical history and physical examination are required. Thus, when clinicians encounter patients with testicular hydrocele, a variety of possible causes must be considered, including testicular or paratesticular tumors, even rare tumors such as mesothelioma of the tunica vaginalis testis.
\end{abstract}

\section{Introduction}

Primary testicular hydrocele is an abnormal collection of fluid in the scrotum, and its incidence rate in men aged $>40$ years of age is $\sim 1 \%$ (1). However, acquired hydrocele is usually

Correspondence to: $\mathrm{Dr} \mathrm{Xu}$ Luo, Department of Urology, Affiliated Hospital of Zunyi Medical College, 149 Dalian Road, Zunyi, Guizhou 563003, P.R. China

E-mail: energy20170118@hotmail.com

Key words: malignant mesothelioma, tunica vaginalis, radical orchiectomy, pemetrexed and cisplatin idiopathic and may be caused by epididymitis and trauma, or, in rare cases, a testicular tumor. Testicular mesothelioma is extremely rare, representing $<5 \%$ of all mesotheliomas $(2,3)$; however, it is an often fatal type of testicular malignancy that originates from mesenchymal tissue and may be incidentally found at the time of hydrocele surgery (4-6). The majority of these cases present with non-specific symptoms, such as a painless scrotal mass associated with hydrocele, even at the advanced stage. The pathogenesis of this malignant neoplasm is unclear and is mostly associated with asbestos exposure (7), as well as long-lasting hydrocele (8). Early preoperative diagnosis may be difficult, and a few cases have even been initially misdiagnosed as epididymitis (6). We herein describe the case of malignant mesothelioma of the tunica vaginalis testis, together with a review of the related literature.

\section{Case report}

A 50-year-old man, who was previously diagnosed with left hydrocele in January 2015, visited the Department of Urology of the Affiliated Hospital of Zunyi Medical College (Zunyi, China) in July 2015, due to painless enlargement of the left scrotum for 6 months, which gradually progressed. On physical examination, the left scrotum appeared to be edematous and had a size of $8 \times 6 \times 5 \mathrm{~cm}$, but there was no palpable mass. Ultrasonography revealed left hydrocele and multiple iso-echoic masses, sized 3-5 mm, located at the inner sheath of the left periorchium. Tunica vaginalis subinvolution was performed, and the postoperative pathological examination revealed malignant mesothelioma arising from the left tunica vaginalis testis. Whole-body positron emission tomography-computed tomography (PET-CT) revealed no evidence of local lymphadenopathy (Fig. 1), and radical left orchiectomy was performed after the pathological diagnosis. Pathological examination after the second surgery confirmed the diagnosis of malignant mesothelioma arising from the left tunica vaginalis testis, and demonstrated that the tumor had invaded the adjacent periorchium and spermatic cord; there was no evidence of local lymph node metastasis.

Histopathological examination revealed a biphasic pattern with mixed epithelial and spindle cell proliferation (Fig. 2). Immunohistochemical examination with a primary rabbit polyclonal anti-vimentin antibody (Santa Cruz Biotechnology, CA, USA) revealed positive staining $(+++)$ for vimentin (Fig. 3) in the epithelial nests, particularly in the spindle cells. 


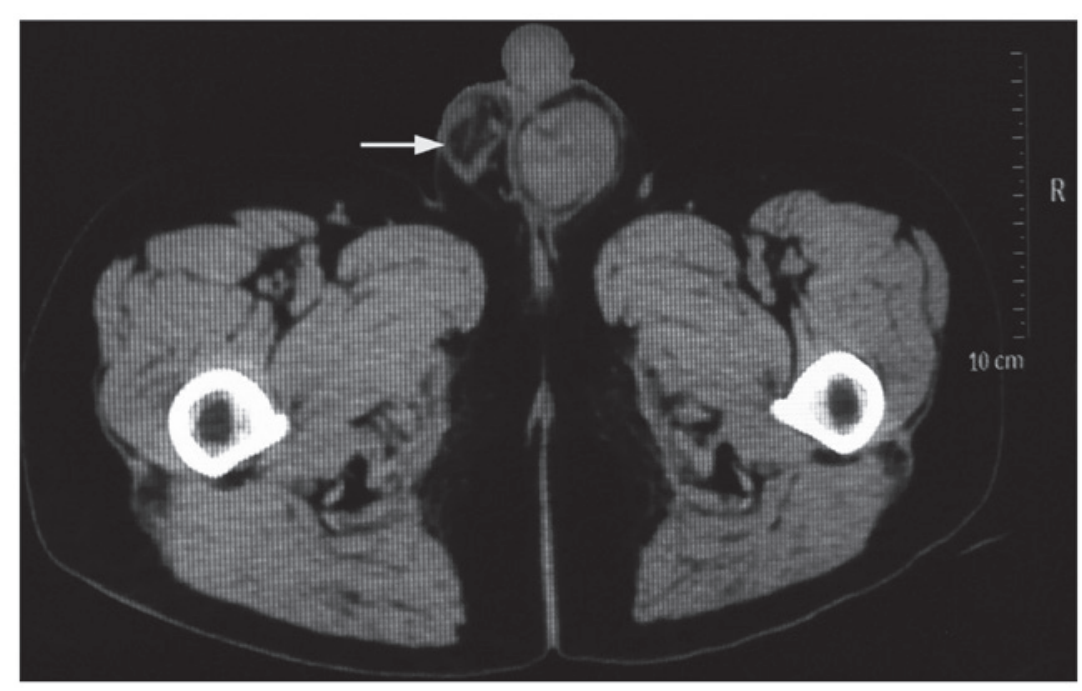

Figure 1. Positron emission tomography-computed tomography of the left testis showing a tumor (arrow) involving the scrotal wall. There was no enlargement of the local lymph nodes.

The postoperative course was uneventful and the surgical incision healed well. Cytotoxic chemotherapy was administered 1 month after surgery: Pemetrexed and cisplatin were administered at a dose of 900 and $130 \mathrm{mg}$, respectively, on the first day of a 28-day cycle. After 6 months of therapy, the disease had not progressed on PET-CT and abdominal and pelvic MRI (Fig. 4). The progression-free survival time was 24 months at the time of the present report, and no local recurrence was detected during the regular follow-up visits (last follow-up visit, April 2017). The patient had no known exposure to asbestos.

\section{Discussion}

Approximately $68-85 \%$ of malignant mesotheliomas originate from the pleura, and 9.1-24.1\% arise from the peritoneum (9). Malignant mesothelioma of the tunica vaginalis testis is an extremely rare occurrence, representing only $0.3-5 \%$ of all mesotheliomas (10). Since the first case of malignant mesothelioma of the tunica vaginalis testis was reported by Barbera and Rubino in 1957 (10), 100 cases have been reported to date. The incidence of malignant mesothelioma is roughly 11 cases per million people annually in the United States (11).

The etiology and mechanism underlying the development of malignant mesothelioma of the tunica vaginalis testis has not been fully elucidated, but exposure to asbestos is a well-known risk factor $(4,11)$, and direct contact with asbestos significantly increases the risk of developing a malignant mesothelioma (12-14). The clinical symptoms of malignant mesothelioma of the tunica vaginalis testis are usually atypical and non-specific, and preoperative diagnosis may be challenging for clinicians, but it should be suspected in any patient with a history of exposure to asbestos presenting with scrotal diseases (15-17). As reported this case, there were no clinical symptoms apart from left testicular hydrocele for $>6$ months. Left scrotal hydrocele was diagnosed and tunica vaginalis subinvolution was initially performed. The pathological diagnosis was malignant mesothelioma arising from the left tunica vaginalis testis. According to the histopathological charac- teristics, malignant mesothelioma has been sub-classified as epithelioid, sarcomatoid and biphasic. The epithelial type is the most common in the peritoneal cavity and the tunica vaginalis, as in the present case (Fig. 2).

In our patient, the left scrotum gradually increased in size, but the parenchyma and size of the left testis were normal. Ultrasonography revealed several nodes of different sizes. Under such circumstances, CT should be performed to stage the tumor, diagnose distant metastases and, particularly, evaluate the pathological status of the retroperitoneal lymph nodes (18). Prior to radical left inguinal orchiectomy, whole-body PET-CT was performed and revealed no evidence of local lymphadenopathy or distant metastasis. In addition to physical examination and imaging studies, it is important to confirm the diagnosis of malignant testicular mesothelioma with pathological examination. Malignant testicular mesothelioma arises from mesenchymal tissue; thus, immunohistochemical staining should be performed to ensure an accurate diagnosis.

Surgery, radiotherapy and chemotherapy are currently the main treatment options for malignant mesothelioma of the tunica vaginalis testis, and comprehensive treatment is often necessary for patients with advanced disease (19). As the disease was localized in the present case, radical inguinal orchiectomy was performed, without retroperitoneal lymphadenectomy. It has been reported that, if any sign of metastasis is evident in the inguinal lymph nodes, inguinal lymph node dissection is required (20). The usefulness of adjuvant chemotherapy and/or radiotherapy has not been clearly demonstrated due to the limited number of reported cases. At present, as cases of malignant mesothelioma of the tunica vaginalis testis are rare, radiation and chemotherapy for malignant testicular mesothelioma remain controversial. Cisplatin and pemetrexed may be used as chemotherapy for testicular mesothelioma (21). In the present case, pemetrexed and cisplatin were administered at a dose of 900 and $130 \mathrm{mg}$, respectively, on the first day of a 28-day cycle for a total of six chemotherapy cycles. After 6 months of therapy, the patient was stable and exhibited no signs of recurrence or metastasis; the patient did not receive radiotherapy, as he was surgically treated at an early stage. 

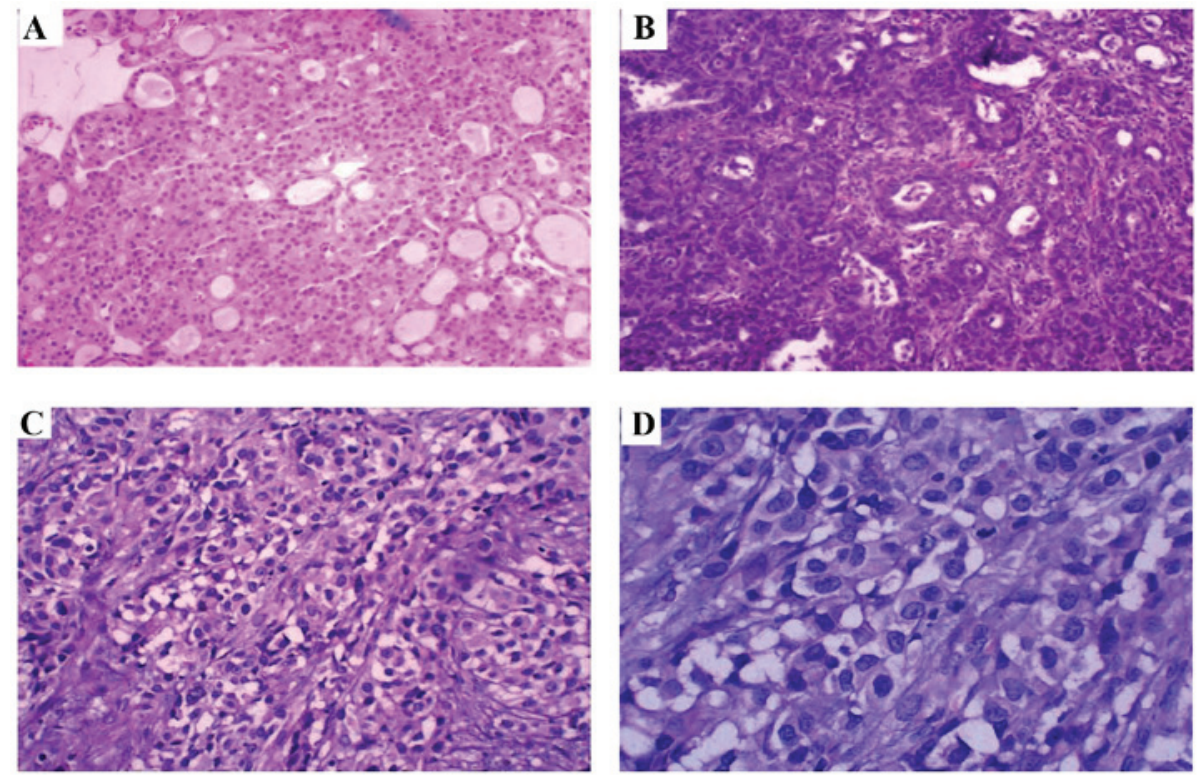

Figure 2. Histological characteristics of malignant mesothelioma of the tunica vaginalis testis. (A) The tumor exhibited a typical epithelioid pattern [hematoxylin and eosin staining (H\&E); magnification, x100]. (B-D) The nuclei of the tumor cells were significantly increased in size and exhibited deep staining. Pathological mitotic figures and a large amount of necrotic tissue were observed (H\&E; magnification, x100, x200 and x400, respectively).

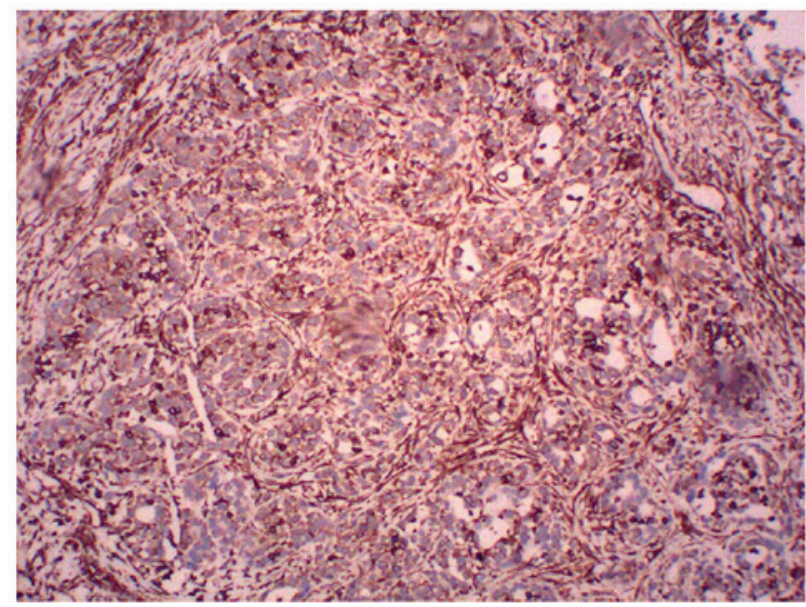

Figure 3. Immunohistochemical examination of malignant mesothelioma of the tunica vaginalis testis revealed strong expression of vimentin (+++). Magnification, $\mathrm{x} 100$
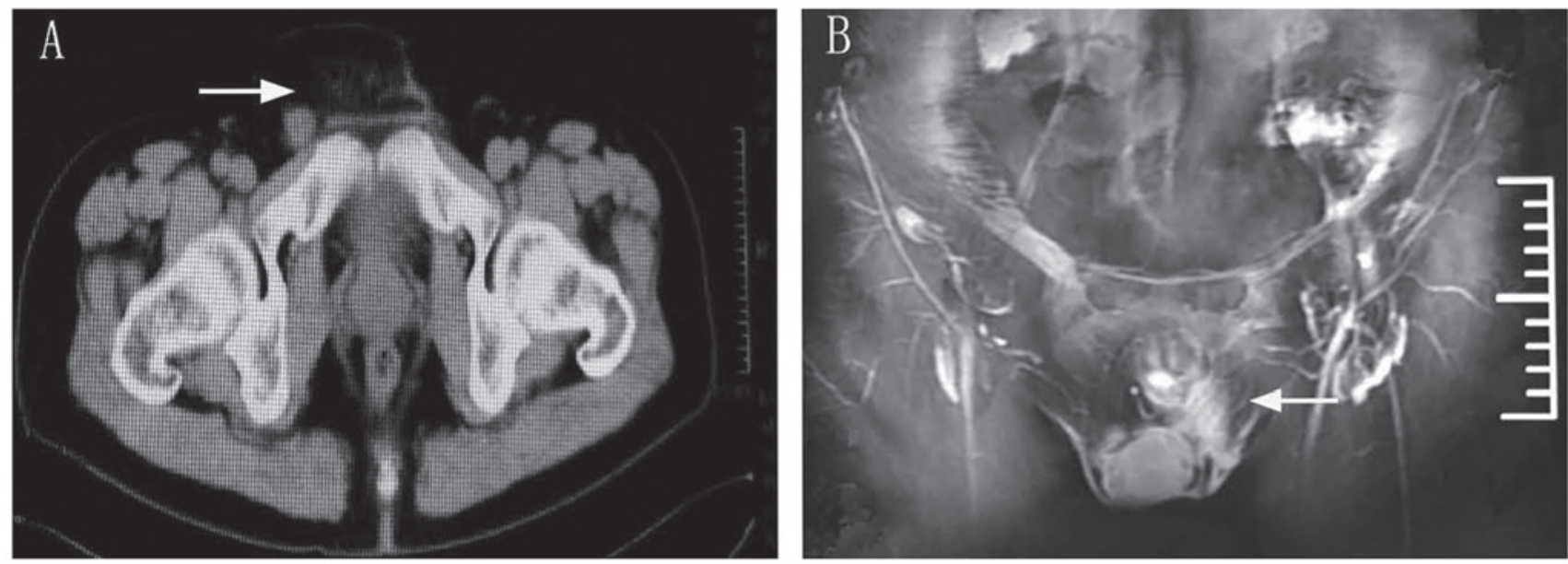

Figure 4. Postoperative positron emission tomography-computed tomography and pelvic magnetic resonance imaging. (A) There were no inguinal (arrow) or retroperitoneal enlarged lymph nodes. (B) Absent left testicle (arrow), without signs of local recurrence. 
The mortality rate from testicular mesothelioma has been reported to be $53 \%$ over a mean follow-up period of 2 years (8). PET-CT and MRI were performed, and revealed no evidence of local recurrence or distant metastasis of tumor. The patient has been followed up at 6-month intervals for the first 2 years in our outpatient clinic, and will continue at 1-year intervals thereafter. The last follow-up visit was in April, 2017.

In summary, diagnosing a primary malignant tumor of the tunica vaginalis testis may be difficult. Physical examination, imaging studies, pathological examination and immunohistochemical staining are required to confirm the diagnosis of malignant testicular mesothelioma. In the present case, chemotherapy (pemetrexed + cisplatin), and long-term follow-up were deemed necessary.

\section{Acknowledgements}

The authors would like to thank the patient for his participation and his consent to the publication of the case details and associated images.

\section{References}

1. Park YJ, Kong HJ, Jang HC, Shin HS, Oh HK and Park JS Malignant mesothelioma of the spermatic cord. Korean J Urol 52: 225-229, 2011

2. Shimada S, Ono K, Suzuki Y and Mori N: Malignant mesothelioma of the tunica vaginalis testis: A case with a predominant sarcomatous component. Pathol Int 54: 930-934, 2004.

3. Chen JL and Hsu YH: Malignant mesothelioma of the tunica vaginalis testis: A case report and literature review. Kaohsiung J Med Sci 25: 77-81, 2009.

4. Antman K, Hassan R, Eisner M, Ries LA and Edwards BK: Update on malignant mesothelioma. Oncology (Williston Park) 19: 1301-1310, 1313-1316, 2005.

5. Murai Y: Malignant mesothelioma in Japan: Analysis of registered autopsy cases. Arch Environ Health 56: 84-88, 2001.

6. Plas E, Riedl CR and Pflüger H: Malignant mesothelioma of the tunica vaginalis testis: Review of the literature and assessment of prognostic parameters. Cancer 83: 2437-2446, 1998.
7. Gupta NP, Agrawal AK, Sood S, Hemal AK and Nair M: Malignant mesothelioma of the tunica vaginalis testis: A report of two cases and review of literature. J Surg Oncol 70: 251-254, 1999.

8. Gürdal M and Erol A: Malignant mesothelioma of tunica vaginalis testis associated with long-lasting hydrocele: Could hydrocele be an etiological factor? Int Urol Nephrol 32: 687-689, 2001.

9. Yang LH, Yu JH, Xu HT, Lin XY, Liu Y, Miao Y, Wang L, Fan CF, Jiang GY, Ding SL, et al: Mesothelioma of the tunica vaginalis testis with prominent adenomatoid features: A case report. Int J Clin Exp Pathol 7: 7082-7087, 2014.

10. Barbera V and Rubino M: Papillary mesothelioma of the tunica vaginalis. Cancer 10: 183-189, 1957.

11. Attanoos RL and Gibbs AR: Primary malignant gonadal mesotheliomas and asbestos. Histopathology 37: 150-159, 2000.

12. Jones MA, Young RH and Scully RE: Malignant mesothelioma of the tunica vaginalis. A clinicopathologic analysis of 11 cases with review of the literature. Am J Surg Pathol 19: 815-825, 1995.

13. Vianna NJ and Polan AK: Non-occupational exposure to asbestos and malignant mesothelioma in females. Lancet 1: 1061-1063, 1978.

14. Ascoli V, Cavone D, Merler E, Barbieri PG, Romeo L, Nardi F and Musti M: Mesothelioma in blood related subjects: Report of 11 clusters among 1954 Italy cases and review of the literature. Am J Ind Med 50: 357-369, 2007.

15. Frank AL: Medical and public health approaches to asbestos disease. Mt Sinai J Med 62: 401-405, 1995.

16. McDonald JC: Health implications of environmental exposure to asbestos. Environ Health Perspect 62: 319-328, 1985.

17. Merler E: Mesothelioma incidence decreases parallel to asbestos exposure decrement or interruption: A confirmation of a dose-response relationship, with implications in public health. Epidemiol Prev 31 (4 Suppl 1): S46-S52, 2007 (In Italian).

18. Yen CH, Lee CT, Su CJ and Lo HC: Malignant mesothelioma of the tunica vaginalis testis: A malignancy associated with recurrent epididymitis? World J Surg Oncol 10: 238, 2012.

19. Yan TD, Welch L, Black D and Sugarbaker PH: A systematic review on the efficacy of cytoreductive surgery combined with perioperative intraperitoneal chemotherapy for diffuse malignancy peritoneal mesothelioma. Ann Oncol 18: 827-834, 2007.

20. Akin Y, Bassorgun I, Basara I and Yucel S: Malignant mesothelioma of tunica vaginalis: An extremely rare case presenting without risk factors. Singapore Med J 56: e53-e55, 2015.

21. Ceresoli GL, Zucali PA, Favaretto AG, Grossi F, Bidoli P, Del Conte G, Ceribelli A, Bearz A, Morenghi E, Cavina R, et al: Phase II study of pemetrexed plus carboplatin in malignant pleural mesothelioma. J Clin Oncol 24: 1443-1448, 2006. 